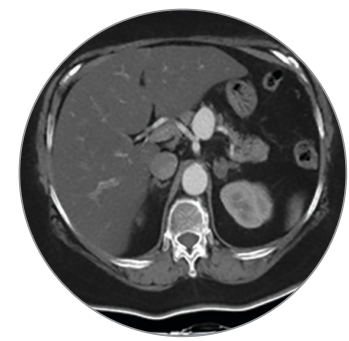

\title{
Aneurisma de arteria esplénica, causa infrecuente de dolor abdominal. Presentación de un caso
}

\author{
Splenic Artery Aneurysm, Uncommon Cause of Abdominal Pain. A \\ Case Report
}

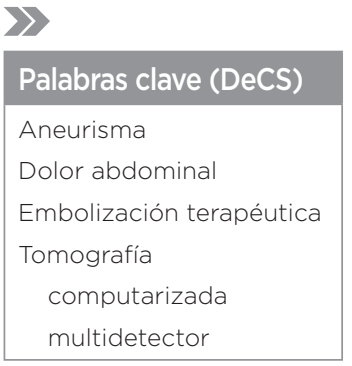

Key words (MeSH)

Aneurysm

Abdominal pain

Embolization, therapeutic

Multidetector computed tomography
Medica radióloga, Neurodinamia. Docente adjunta Departamento de Radiología, Universidad de Cartagena. Cartagena, Colombia.

${ }^{2}$ Residente de Radiología, Universidad de Cartagena. Cartagena, Colombia.

${ }^{3}$ Médico. Universidad de Cartagena. Cartagena, Colombia.

\section{Resumen}

Los aneurismas de las arterias viscerales son relativamente poco frecuentes. El aneurisma de la arteria esplénica es el más común de este grupo, se presenta en el 60 a 70 \% de los pacientes. Presentamos el caso de una paciente de 68 años de edad con factores de riesgo cardiovascular, quien consultó por un cuadro de dolor abdominal agudo, intenso, asociado a sensación de peso, y masa pulsátil al examen físico como dato representativo. Mediante angioTAC abdominal y angiografía de tronco celiaco se diagnosticó una dilatación aneurismática sacular en el segmento proximal de la arteria esplénica, la cual, por su localización y con el objetivo de preservar el bazo, fue tratada mediante cateterización selectiva y embolización del saco del aneurisma, para reducir el riesgo de ruptura y lograr un control de síntomas adecuado. A continuación, se explica brevemente la fisiopatología, diagnóstico y los beneficios de la terapia endovascular respecto a otras técnicas.

\section{Summary}

Visceral artery aneurysms are relatively rare. The splenic artery aneurysm is the most common in this group, occurring in 60 to $70 \%$ of patients. We present the case of a 68 year old patient with cardiovascular risk factors, who consulted for acute, intense abdominal pain associated with sensation of weight, and a pulsatile mass at physical examination as representative data. Diagnosis of an saccular aneurysmal dilation in the proximal segment of the splenic artery was made by abdominal angiography and celiac trunk angiography, which, due to its location and to preserve the distal flow, was treated by selective catheterization and embolization of the aneurysm sac, thus reducing risk of rupture and achieving clinical control of the patient's symptoms. The physiopathology, diagnosis, and benefits of endovascular therapy compared to other techniques are briefly explained below.

\section{Introducción}

El aneurisma de la arteria esplénica se define como una dilatación anormal de más del $50 \%$ de diámetro del vaso, es el tercer aneurisma abdominal más frecuente después del aneurisma de la aorta y arterias ilíacas $(1,2)$. Hace parte de los aneurismas de arterias viscerales, con una incidencia del $60 \%$ (3). El diagnóstico oportuno es de mucha importancia por el riesgo de ruptura y hemorragia potencialmente mortal. A menudo, el diagnóstico en fases iniciales es incidental, debido a que en esta etapa es asintomático y, de presentarse, la sintomatología es muy inespecífica (4).

\section{Caso clínico}

Paciente femenina de 68 años de edad, multípara, con antecedentes de hipertensión arterial (HTA), diabetes mellitus (DM) tipo II, hipotiroidismo y aneurisma de la aorta abdominal en vigilancia activa. Acude al servicio de urgencias por cuadro clínico de
3 días de evolución, consistente en dolor abdominal en epigastrio de intensidad 10/10 según escala análoga de dolor, con sensación de masa e hipersensibilidad local. $\mathrm{Al}$ examen físico de ingreso se encontró hemodinámicamente estable, afebril, abdomen blando, percusión y peristaltismo adecuado, hipersensibilidad en hemiabdomen superior y, a la palpación, masa pulsátil en el epigastrio. Se realizó inicialmente una AngioTAC de abdomen con medio de contraste en la que se observó dilatación sacular de arteria esplénica proximal a $1 \mathrm{~cm}$ de su nacimiento (figura 1).

Así mismo, mediante angiografía del tronco celiaco se evidenció una formación aneurismática sacular a 10 $\mathrm{mm}$ del origen de $25 \times 30 \mathrm{~mm}$ con ancho de cuello de $1 \mathrm{~mm}$ en el tercio proximal de arteria esplénica.

Teniendo en cuenta los riesgos quirúrgicos y la localización anatómica de la lesión se consideró realizar terapia endovascular mediante embolización con espirales (coils) consistente en: canalización de aneurisma sacular y liberación interlook detacheable coil $4 \mathrm{~mm} \times$ 
$8 \mathrm{~cm}$, detacheable coil $2 \mathrm{~d}: 10 \mathrm{~mm} \times 20 \mathrm{~cm}, 12 \mathrm{~mm} \times 20 \mathrm{~cm}, 15 \mathrm{~mm} \times$ $20 \mathrm{~cm}$. Después del procedimiento se tomó una angiografía de control que evidenció exclusión completa del aneurisma, no se presentaron complicaciones inmediatas, por lo que se decidió dar egreso con manejo de sus comorbilidades (figura 2).
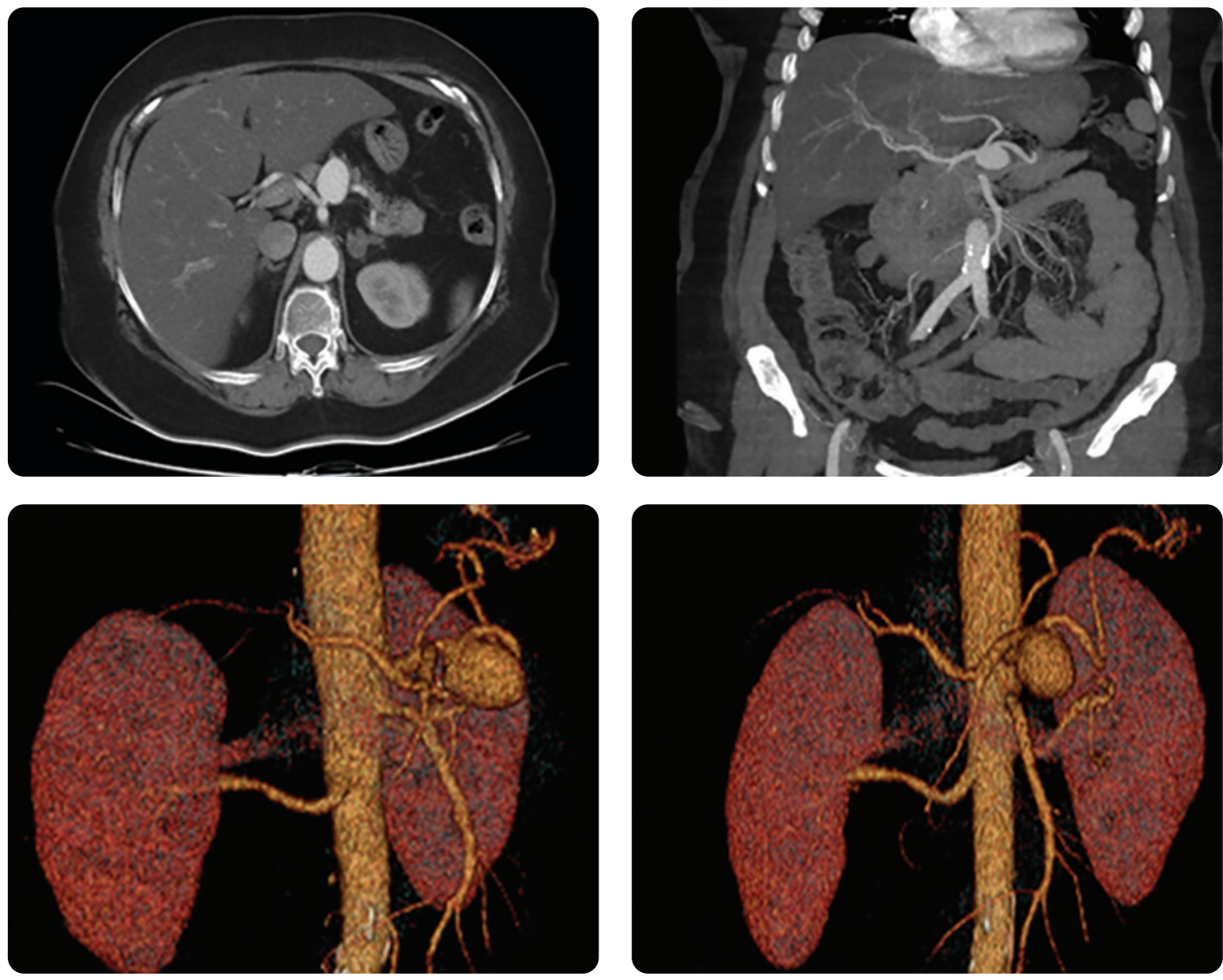

Figura 1. AngioTAC de abdomen a) Corte axial: dilatación sacular de arteria esplénica proximal a 1 $\mathrm{cm}$ de su nacimiento. b). Corte coronal: dilatación sacular de 25 $\times 30 \mathrm{~mm}$, a $10 \mathrm{~mm}$ del origen de la arteria esplénica, de $25 \times$ $30 \mathrm{~mm}$ y con cuello de $1 \mathrm{~mm}$ en el tercio proximal de la arteria esplénica. c y d) Reconstrucción tridimensional.
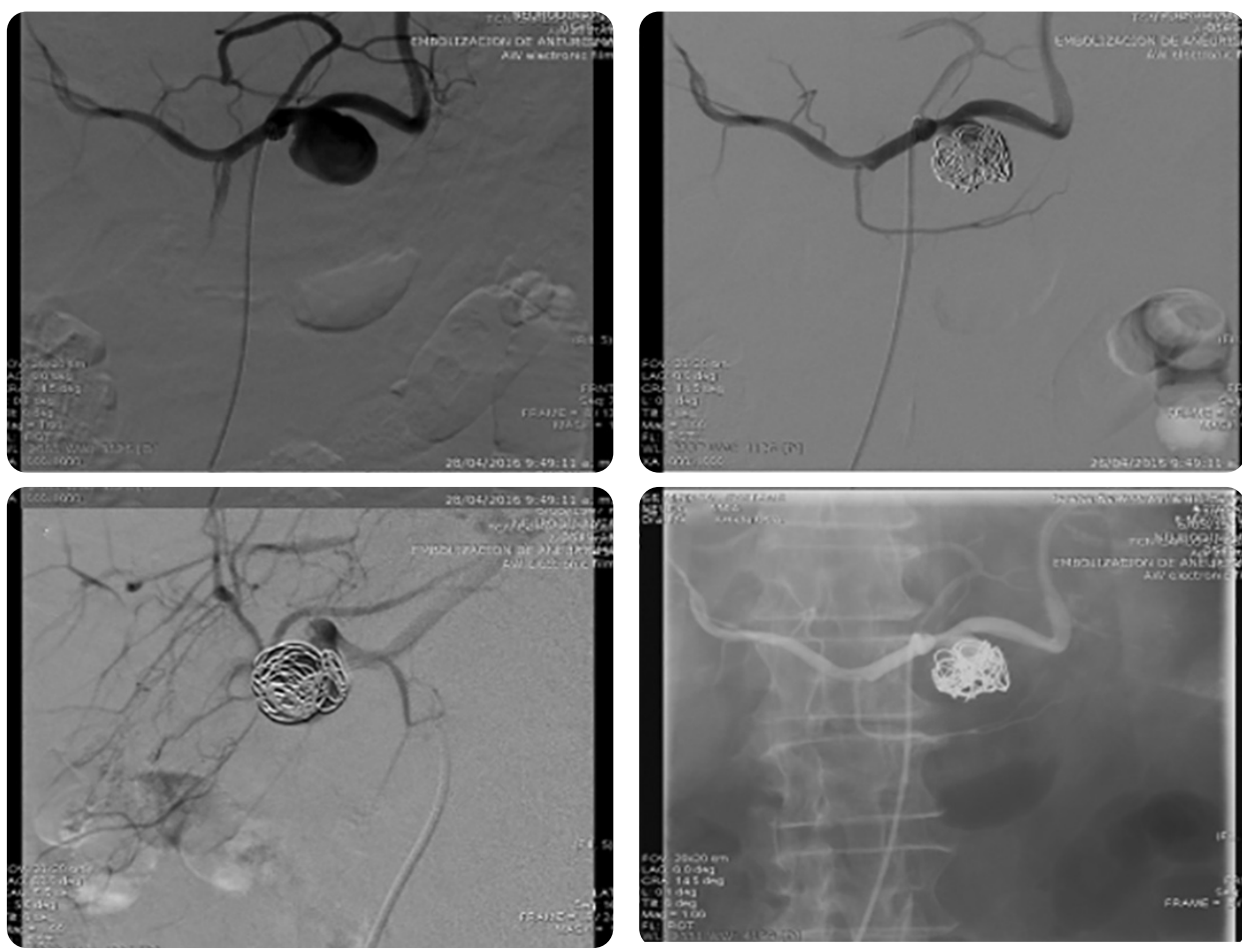

Figura 2. Arteriografía selectiva de la arteria esplénica con sustracción digital:

a) Se observa dilatación sacular de arteria esplénica proximal a $1 \mathrm{~cm}$ de su nacimiento. b y c) Control postembolización con espirales, que muestra la adecuada oclusión del cuello del aneurisma. d) Control postembolización con espirales de aneurisma. 


\section{Discusión}

Los aneurismas de las arterias viscerales tienen una incidencia de 0,1 a $0,2 \%$ y en la arteria esplénica en el 60 a $70 \%$ de los casos (4). Son más comunes en mujeres con una proporción $4: 1$, pero son tres veces más propensos a ruptura en hombres (5). El $80 \%$ se producen en pacientes mayores de 50 años, se asocia en la mayoría de los casos a ateroesclerosis (32\%) y a enfermedades crónicas como hipertensión y diabetes, presentes en el caso en discusión. Se incluyen también como facilitadores aquellos factores que aumenten el flujo esplácnico, como las fístulas arteriovenosas y la hipertensión portal, con una prevalencia del $7 \%$ al $20 \%(6,7)$.

Los aneurismas verdaderos de la arteria esplénica son generalmente solitarios, saculares y localizados principalmente en el tercio distal y medial del vaso sanguíneo (8), con mayor frecuencia en la región de la bifurcación e hilio esplénico y su tamaño varía entre los 2 y $9 \mathrm{~cm}(4,9)$.

Son asintomáticos en un $97 \%$ de los pacientes, por lo que su identificación es incidental en estudios de imagen (10). La ruptura es la principal complicación debido a la hemorragia potencialmente mortal que se genera, teniendo en cuenta que los aneurismas gigantes $>2,5$ $\mathrm{cm}$ elevan el riesgo de ruptura hasta un $40 \%$ (4).

Clínicamente se presentan como un cuadro de abdomen agudo, asociado a hematoquecia o melena en un $26,2 \%$ y hematemesis hasta un $14,8 \%$. La mortalidad varía entre 10 y $25 \%$ en pacientes no embarazadas, y en gestantes puede llegar a un $70 \%$ (3).

Las imágenes diagnósticas tienen un papel importante. El ultrasonido se usa comúnmente en la detección de los aneurismas aórticos abdominales con transductores de baja frecuencia (por ejemplo, 4 $\mathrm{MHz}$ ) realizando análisis Doppler y escala de grises, lo que permite estimar el tamaño vascular y las velocidades de flujo interno (11). Esta técnica permite detectar aneurismas de la arteria esplénica con la misma precisión como se ve en los aneurismas aórticos (12). Es limitado por gas intestinal, lo que puede imposibilitar la visualización los vasos retroperitoneales y mesentéricos (11), pero mejora su rendimiento con la previa preparación del paciente.

La TC desempeña un papel más importante en la confirmación del diagnóstico y caracterización adicional de aneurismas y pseudoaneurismas viscerales. La TC multidetector con adquisiciones en distintas fases puede mostrar con precisión y claridad la ubicación, forma, extensión, pared aneurismática, arteria principal y la relación con los vasos adyacentes (13).

Las imágenes angiográficas de TC en fase arterial se obtienen típicamente 20-30 segundos después del inicio de la inyección intravenosa periférica a una velocidad de 3-4 mL/s. Su uso es esencial, porque puede confirmar el diagnóstico de un aneurisma visceral roto y utilizarse para planificar el procedimiento endovascular (11).

La información incluye:

- Determinar el plan terapéutico: tipo de abordaje (femoral, humeral), tortuosidad de los vasos, y el ángulo de las arterias viscerales respecto a la aorta.

- Número de ramas aferentes y eferentes.

- Las reconstrucciones de representación de volumen (VR, por las iniciales en inglés de rendered volume) y proyección de intensidad máxima (MIP) son útiles para obtener un análisis óptimo del aneurisma.
Por ser una patología poco frecuente y frente a la ausencia de estudios controlados, el tratamiento se basa en los estudios observacionales disponibles, por lo tanto, las indicaciones de tratamiento en pacientes sintomáticos siempre será la reparación sin importar otras características (3). Entre las indicaciones de intervención en paciente asintomático se encuentran: tamaño mayor a $2 \mathrm{~cm}$, crecimiento documentado del aneurisma, pacientes cirróticos, con trasplante hepático, sometidos a cirugía abdominal mayor y embarazadas durante el primer y segundo trimestre de la gestación (14).

Las opciones de tratamiento para los aneurismas de la arteria esplénica incluyen reparación quirúrgica y manejo endovascular. La reparación quirúrgica o la resección del aneurisma con posible esplenectomía es una opción de tratamiento de segunda línea en la mayoría de los casos, aunque, a menudo se rechaza debido al mayor riesgo a largo plazo de infecciones bacterianas. El tratamiento endovascular de elección es la embolización con un agente embolizante líquido y/o espirales - generalmente reservado para aneurismas saculares y aquellos con flujo adecuado o alternativo al bazo- $(13,15,16)$. Para los aneurismas fusiformes, en los que la luz arterial requiere preservación para evitar daño en órgano distal (bazo), se coloca un estent si la anatomía arterial es favorable (16-19).

En la mayoría de estudios, la reparación y reconstrucción quirúrgica abierta sigue siendo la estrategia óptima en pacientes hemodinámicamente inestables, politraumatizados o con obstrucción de la vena porta extrahepática (20). En casos de ruptura aneurismática, o aneurismas que implican el hilio esplénico, comúnmente se someten a esplenectomía $(3,10,21)$.

En la actualidad, la vía endovascular cobra un papel protagónico en el manejo de esta patología debido a sus elevadas tasas de éxito y baja morbimortalidad, con buenos resultados en pacientes de cirugía no urgente y candidatos quirúrgicos de riesgo que presentan comorbilidades asociadas o en pacientes con abdomen hostil por pancreatitis, sepsis o cirugías previas. Las principales ventajas de esta técnica son la baja tasa de complicaciones, disminución del dolor postoperatorio, reducción de la estancia hospitalaria, retorno más rápido a las actividades cotidianas y mejor calidad de vida a corto plazo $(2,14,22)$.

La mayoría de los procedimientos endovasculares (80-90 \%) son técnicamente exitosos, con solo un pequeño grado de infarto esplénico. El flujo colateral, sobre todo a través de las arterias gástricas cortas, mantiene clásicamente la perfusión del órgano terminal. Sin embargo, los riesgos de infarto esplénico aumentan con más embolizaciones distales (11).

Se ha propuesto un seguimiento de imagen a intervalos de 1 año debido a un riesgo del $20 \%$ de reperfusión después de una embolización exitosa. Si se produce reperfusión, el saco vuelve a estar expuesto a presiones sistémicas y, una vez más, puede estar en riesgo de ruptura (16).

\section{Conclusión}

Los aneurismas de la arteria esplénica en su tercio proximal son poco frecuentes. Su diagnóstico suele ser incidental en estudios imagenológicos con medio de contraste, ya que suelen ser asintomáticos. El abordaje quirúrgico aumenta el riesgo de complicaciones, por lo que el manejo endovascular constituye una técnica segura y efectiva para su tratamiento, con bajas tasas de complicaciones y recurrencia. 


\section{Referencias}

1. Kukliński A, Batycki K, Matuszewski W, Ostrach A, Kupis Z, Legowik T. Embolization of a large, symptomatic splenic artery pseudoaneurysm. Polish J Radiology. 2014;79:194-8.

2. Algudkar A. Unruptured splenic artery aneurysm presenting as epigastric pain. JRSM Short Reports. 2010;1(3):1-3.

3. Termos S, Taqi A, Hayati H, Alhasan AJMS, Alali M, Adi A. Segmental arterial mediolysis with 5 splenic artery aneurysms. A rare finding of a rare disease: Case report and literature review. Int J Surg Case Reports. 2017;33:158-62.

4. Al Jalbout N, Moreland AJ. Syncope in a middle aged female: Splenic artery aneurysm revisited. Clinical Imaging. 2018;52(2018):8-10.

5. Etezadi V, Gandhi RT, Benenati JF, Rochon P, Gordon M, Benenati MJ, et al. Endovascular treatment of visceral and renal artery aneurysms. J Vascular Intervent Radiol. 2011;22(9):1246-53.

6. Sachdev-Ost U. Visceral artery aneurysms: Review of current management options. Mt Sinai J Med. 2010;77(3):296-303.

7. Wernheden E, Brenøe A-S, Shahidi S. Emergency endovascular coiling of a ruptured giant splenic artery aneurysm. J Vascular Surg Cases Innovative Techniques. 2017;3(4):240-2.

8. Zhang HY, Chai DZ. Endovascular coil embolization for a giant anomalous splenic artery aneurysm. J Vascular Surg Cases. 2015;1(2):141-3.

9. Zeng D-B. Abnormal splenic artery diameter/hepatic artery diameter ratio in cirrhosisinduced portal hypertension. WJG. 2013;19(8):1292.

10. Abbas MA, Stone WM, Fowl RJ, Gloviczki P, Oldenburg WA, Pairolero PC, et al Splenic artery aneurysms: Two decades' experience at Mayo Clinic. Ann Vascular Surg. 2002;16(4):442-9.

11. Jesinger RA, Thoreson AA, Lamba R. Abdominal and pelvic aneurysms and pseudoaneurysms: Imaging review with clinical, radiologic, and treatment correlation. RadioGraphics. 2013;33(3):E71-96.

12. Piasek E, Drelich-Zbroja A, Sojka M, Pyra K, Kuczyńska M, Szczerbo-Trojanowska M. Ultrasound imaging of splenic artery aneurysms. Post N Med. 2017;30(04): 210-2.

13. Zeman RK, Baron RL, Jeffrey RB, Klein J, Siegel MJ, Silverman PM. Helical body CT: evolution of scanning protocols. Am J Roentgenol. 1998;170(6):1427-38

14. Parrish J, Maxwell C, Beecroft JR. Splenic artery aneurysm in pregnancy. J Obstetrics Gynaecol Canada. 2015;37(9):816-8.

15. Tulsyan N, Kashyap VS, Greenberg RK, Sarac TP, Clair DG, Pierce G, et al. The endovascular management of visceral artery aneurysms and pseudoaneurysms. J Vascular Surg. 2007;45(2):276-83.

16. Sachdev U, Baril DT, Ellozy SH, Lookstein RA, Silverberg D, Jacobs TS, et al. Management of aneurysms involving branches of the celiac and superior mesenteric arteries: A comparison of surgical and endovascular therapy. J Vascular Surg. 2006;44(4):71824.

17. Madoff DC, Denys A, Wallace MJ, Murthy R, Gupta S, Pillsbury EP, et al. Splenic arterial interventions: Anatomy, indications, technical considerations, and potential complications. RadioGraphics. 2005;25(Suppl-1):S191-211.

18. Arepally A, Dagli M, Hofmann LV, Kim HS, Cooper M, Klein A. Treatment of splenic artery aneurysm with use of a stent-graft. J Vascular Intervent Radiol. 2002;13(6):631-

19. Larson RA, Solomon J, Carpenter JP. Stent graft repair of visceral artery aneurysms. J Vascular Surg. 2002;36(6):1260-3.

20. Mishra PK, Saluja SS, Sharma AK, Pattnaik P. Management of splenic artery aneurysm associated with extrahepatic portal vein obstruction. Hepatob Pancreatic Dis Internat. 2012;11(3):330-3.

21. Hogendoorn W, Lavida A, Hunink MGM, Moll FL, Geroulakos G, Muhs BE, et al Cost-effectiveness of endovascular repair, open repair, and conservative management of splenic artery aneurysms. J Vascular Surg. 2015;61(6):1432-40.

22. Gehlen JMLG, Heeren PAM, Verhagen PF, Peppelenbosch AG. Visceral artery aneurysms. Vasc Endovascular Surg. 2011;45(8):681-7.

\section{Correspondencia}

Micaela Arrieta Usta

Universidad de Cartagena

Centro Carrera 6 \# 36-100, Claustro San Agustín

Cartagena, Colombia

micaelarrieta@gmail.com

Recibido para evaluación: 10 de febrero de 2020

Aceptado para publicación: 5 de abril de 2020 